\title{
FDIs and Commercial Balance in CEE Countries - Special Focus on the Manufacturing Economic Sectors. A VAR Analysis
}

\author{
Radulescu Magdalenaํㅡ, Cirstea Cornelia Gabriela ${ }^{2}$, Belascu Lucian Aron² \\ ${ }^{\prime}$ University of Pitesti, Department of Finance, Accounting and Economics \\ "University "Lucian Blaga" of Sibiu, Department of Management, Marketing and Business Administration
}

\begin{tabular}{l}
\hline ARTICLE INFO \\
\hline Article History \\
Received 03 August 2020; \\
Accepted 08 September \\
2020 \\
\hline JEL Classifications \\
F14, F21, L60.
\end{tabular}

\begin{abstract}

Purpose:

Some empirical findings of the role of foreign direct investment (FDIs) in a host country's export performance was found by many researchers, since exports have been for a long time viewed as an engine of economic growth. But exports and imports are inter-correlated and some works proved that sometimes, the foreign-owned companies import more than they export in some economic sectors. The paper aims to establish the relation between total FDIs and the commercial balance (goods) and between FDI stocks in the manufacturing economic sectors and the commercial balance of manufactured goods in 11 Central and Eastern European countries during the crisis period and post-crisis period (2009-2018). We have tested the causality using Granger causality test to see if there is a uni-directional or bi-directional causality between those variables. We have tested for co-integration and we haven't found a long-term relationship between those variables and we have applied the VAR technique. Our results have proved a bi-directional causality between FDI stockexports-imports and a stronger impact of FDIs stock on the trade balance of manufactured goods than the impact of total FDI stock on the commercial balance of goods in CEE
\end{abstract} countries.

Design/methodology/approach:

Granger causality tests, cointegration test, VAR analysis

Finding:

We have found no cointegration in the long-run between FDIs stocks and exports and imports in the CEE countries. We have found a bi-directional causality between those variables. We have found a stronger impact of FDIs stock on export and imports of manufactured goods than the impact of FDIs stock on total exports and imports in the CEE

Keywords: commercial balance, manufactured goods, FDI stock,

CEE countries.

\section{Research limitations/implications:}

This research can be extended analysing a longer period of time and including more exogenous variables in the analysis such as labour productivity, labour cost and GDP growth. It can also be performed a panel analysis. The CEE countries should design adequate policies in order to attract more FDIs in the manufacturing sectors, given the strong impact of FDI stock for these sectors and given the large share of the manufactured goods of the total exports of the CEE countries.

Originality/value:

This research is important for CEE region because of the large share of the manufactured good of the total exports of these economies.

\footnotetext{
1. Introduction behaviour.

${ }^{\dagger}$ Corresponding Author: RADULESCU MAGDALENA

Email: Magdalena.radulescu@upit.ro
}

Foreign Direct Investment (FDI) plays a pivotal role in supporting the development of countries' economic development process (Raeskyesa and Suryandaru, 2020). FDI was the principal source of flows to the developing countries in 1990. Unlike other capital flows, FDI has a fewer degree of volatility and does not follow a pro-cyclical 
The simulative effects of FDI on exports of the host country come from the additional capital, technology and managerial know-how of the multinational corporations, but also from the access to global, regional and homecountry markets (UNCTAD, 2002).

By processing components and assembling the imports of the unfinished and intermediate goods, China became a dominant exporter of labour-intensive products (toys, shoes, clothes and sporting goods) and some technologyintensive products (machinery and equipment, including electronic circuits, automatic data-processing machines and mobile phones) (UNCTAD, 2002). Many studies have demonstrated that growth in the manufacturing sector is a key driver in the economic growth in China (Haruchi, Smeet, \& Chen, 2017). Using a regression analysis, Karanja (2019) found there is a very high correlation between the level of FDI and manufacturing industry productivity in China and, thus, China needs to attract more FDIs. Not only China developed a lot based on the export's growth of the manufactured goods. Another „Asian Tigers” (in terms of economic growth) greatly developed as a result of large FDI inflows and important exports growth of the manufactured goods. Many previous studies focused on „Asian Tigers" and, especially, on China.

Sekkat (2015) found that deeper integration with the EU area increased manufactured exports and FDIs to a greater degree compared to other cases of the regional integration.

Krstić (2014) has shown that economic models allow us to improve the level of understanding of the economy and not only of the economy.

This paper aims to find out what is the correlation between the FDIs stock and total exports and imports in the CEE countries and what is the correlation between FDI stock in the manufacturing sectors and exports and imports of the manufactured goods in this specific region that attracted large FDI inflows in the 2000 ' and reached high economic growth rates (based on exports) comparing to the rest of EU countries. Of course, the positive correlation between the FDI inflows and exports growth of the manufactured goods depends on the productivity in the manufacturing sectors and on the level of the domestic supply on the internal market. An insufficient domestic supply or a low productivity in the manufacturing sectors can't support important positive spillovers of FDIs on exports or an increase of the exports of the manufactured goods.

There are many studies focused on the relation between FDI stock and total exports growth, but only few of them have dealt with European countries or with the Central and Eastern European countries and have studied the relation between FDI and exports and imports in the same time.

\section{Literature Review}

Camarero and Tamarit (2004) studied the relationship between foreign trade with manufactured products (both exports and imports) and FDI inflows and outflows for 11 European Union countries, USA and Japan using quarterly data between 1981 and 1998. The authors found a positive and significant relationship between FDI and foreign trade.

Damijan et al. (2008) studied the export performance in the CEE countries, focusing on the transition countries. The authors found that higher levels of FDI contributed to increasing exports, due to their involvement in restructuring the manufacturing sector.

A GMM analysis for EU countries during 1999-2012 (Popovici, 2018) found that the foreign investments seem to have a higher impact on increasing exports in the new EU member states than in the old ones, irrespective of the economic sector. Instead, in the old EU member states, the domestic investment is the main factor of the export development. Both foreign and domestic investments have a positive impact on the exports. More exports in manufacturing or services will draw a higher volume of exports in the following year. Their previous trend is more important than the developments of the domestic or foreign investments. The exports in the manufacturing sector are more sensitive to the impact of foreign investments than the service sectors that depend more on the domestic investments.

Zaman et al. (2011) have demonstrated in their research that in Romania only FDI inflows in the manufacturing industries (cars industry and metallurgy) and in the agriculture sector generated positive results for the commercial balance, while FDI inflows in the commerce sector and extraction industry generated some high negative results for the Romanian external trade. This can be explained by a high share of imports with high added value in those sectors and an important share of exports with low added value and very low processed. Other researchers (Mishal şi Abulaila, 2007) have stressed a bi-directional relation between FDI, exports and imports. Some other studies have demonstrated that, on average, the impact of FDI on exports and imports is negative, meaning that the foreign-owned firms import more than they export (Borensztein E. et al., 1998).

Bouras and Raggad (2015) have stressed whether export and FDI substitute or complement each other for 10 countries in Africa and Europe. Their random effect model used data for the manufacturing and non-manufacturing sectors in ten developing and non-developing countries. The results show there is a complementarity effect between total exports and total FDI. Similar results were found between manufacturing exports and manufacturing FDI and between non-manufacturing exports and non-manufacturing FDI. The estimated results show that exports have a significant positive impact on FDI. The results show that the complementary effect on exports is predominant at the macro level for manufactured exports relative to non-manufacturing exports. Moreover, if FDI is mainly directed towards the exploitation of natural resources, they should lead to more concentrated export (oil-exporting countries). Results suggest that exports in these countries generate additional FDIs flows from investing countries (a complementary relationship). 
Selimi et al. (2016) analysed empirically the foreign direct investments and exports performance during the period of 1996-2013 in 9 Western Balkan countries (former-Yugoslavia countries and Albania, Romania and Bulgaria). The paper also investigates for the fixed effects and individual heterogeneity across countries and years. Based on the panel regression techniques, Least Square Dummy Variable (LSDV) regression method and a pooled OLS, the authors proved that FDIs positively affect export performance. They have used as exogenous variables FDI, real exchange rate, real GDP growth, added value in the industry, savings and gross capital stock. The fixed effects of FDI on exports are higher in Slovenia, Bulgaria and Macedonia, while they are lower in other 4 SEE countries and even negative in Albania and Serbia.

Jevcak, Suardi and Setzer (2010) analysed FDI inflows in 10 new EU member countries (EU enlargement from 2004). They found that FDI in the mentioned countries does not have higher contribution to productivity growth and export potential.

Mitic si Ivic (2016) have proved a positive relation between FDI and total exports (1993-2013) in the last two decades for $11 \mathrm{CEE}$ countries (demonstrating a strong relation in some CEE countries such as Bosnia and Herzegovina, Bulgaria, Czech Republic and Slovakia) and between FDI and exports of manufactured goods based on high technology (1996-2012) in the European transition countries (a very strong relation in countries such as Polonia and Slovenia), using a correlation analysis (Pearson correlation coefficient) and including one lag in their analysis. They stressed a tighter correlation between FDI and exports based on high technology than the correlation between total FDI and total exports for the CEE region.

There is evidence of a positive relationship between FDI, the level of specialization and changes in export structure in favour of products with higher value added (Lovrinčević et al., 2004). EU membership was a key factor for attracting FDI and for foreign trade development of countries in transition, including the shift of exports towards higher stages of production- higher added value products (Kaminski, 2000). In an analysis of Mitic (2009) during 1994-2009, including 15 European transition countries, the FDI inflows and exports are positively correlated and complementary; the correlation coefficients has slightly higher values than in the case of the relationship between FDI and GDP. A negative correlation that was found in the case of Bulgaria and Croatia (Mitic, 2009). Regarding the impact on industrial exports, the results of an UNCTAD study indicate there is a positive and significant correlation between FDI and industrial exports. The conclusion of this study is that the impact of FDI is more obvious on the technology intensive exports (UNCTAD, 1999).

Anghelache (2016) has demonstrated a linear relation between exports and FDI in Romania using correlogram with a dataset between 1990 and 2015. The linear regression results stressed a positive relation between FDI and exports developments.

Kostoska and Mitrevski (2008) showed in their study for 9 CEE countries during 1985-2006 that the low-income CEE transition economies have developed more slowly comparing to the early reformers in the CEE area (Czech Republic, Hungary and Poland) which have received almost $60 \%$ of the total FDI inflows. The analysis suggests that there have been few or no important positive productivity spillovers of FDI in the analysed transition CEE economies. In other words, the foreign owned firms determined a low productivity growth for the local firms, because of the impulsive domestic competition for the foreign firms and the lack of financial possibility for local firms to adapt well. FDI concentration into non-tradable sector in some CEE countries undermines the export competitiveness. The empirical findings suggest that countries which have attracted more FDI in manufacturing sectors have reached an improvement in export competitiveness against those that attracted FDI flows in the services area $(70 \%$ of manufacturing sales in Hungary were due to foreign investment in late `90s, so the country tripled the exports at the beginning of the following decade). The FDI composition in Czech Republic was divided between services and manufacturing sectors, thus the exports growth was weaker than the increase of the FDI inflows. The same situation was in Bulgaria where manufacturing sectors attracted $1 / 3$ of FDIs, while in Hungary, the foreign owned firms largely participated within the Hungarian exports. The premature reformers such as Poland, Hungary and Czech Republic have allowed a high technology transfer while receiving the most FDIs in the manufacturing sectors. They have increased the $\mathrm{R} \& \mathrm{D}$ composition of their exports, even they still produced low added value products in the high technology sectors. Croatia also faced smaller effects on exports competitiveness, despite large FDI inflows in this country, because the FDIs were attracted mainly in retail and financial intermediation, so the export structure didn't change widely and immediately (there were low technology transfers and less changes in value added composition of their exports). Kersan-Skabic and Zubin (2009) found no significant effect of FDI on exports for the Croatian economy, because of a low share of greenfield investments in Croatia.

Mahmoodi and Mahmoodi (2016) examined the causality relationship between FDI, exports and economic growth in eight European developing countries for 1992 through 2013, and eight Asian developing countries over 1986 to 2013. Panel unit root tests indicated that all variables are I(1) integrated. Panel co-integration tests proved the existence of co-integration in both panels of European or Asian countries. The Panel-VECM causality was performed for both panels, which indicated a unidirectional causality from GDP and FDI to exports in the short-run for the European developing countries. There is evidence of long-run causality from economic growth and export to FDI for both of the European and Asian developing panels. Countries in the two considered panels, especially the European ones, can stimulate the economic growth by attracting FDI inflows. Moreover, countries of these two panels, especially Asian developing countries, can reach higher economic growth by increasing exports of goods and services (by decreasing the export taxes and trade barriers, encourage the industrial-based export and improve quality control). 


\section{FDI inward stock and exports/imports developments in the CEE countries}

If we consider data in Table 1, we can see that in Bulgaria and Croatia, exports increased until 2012 and after that they decreased until 2016. In the last analysed years, they have increased again at similar level of the year 2012 when they reached their peak during the entire analysed period. The same situation can be seen in Estonia, Latvia and Lithuania. For Czechia there was a steady increase almost the entire analysed period of time (except two years of a slow decrease) and the same trend can be observed in Hungary, Poland, Slovakia and Slovenia.

Table 1. Export unit value index $(2015=100)-$ total products, all countries of the world

\begin{tabular}{|c|c|c|c|c|c|c|c|c|c|c|}
\hline & $9 \begin{array}{ll}200 \\
\end{array}$ & $10^{20}$ & $\begin{array}{ll} & 201 \\
1 & \\
\end{array}$ & 2012 & 2013 & $\begin{array}{r}201 \\
4 \quad \\
\end{array}$ & 2015 & 2016 & $\begin{array}{ll} & 201 \\
7 & \\
\end{array}$ & $8^{201}$ \\
\hline $\begin{array}{ll} & \text { Bulgari } \\
\text { a } & \\
\end{array}$ & 85,4 & $\begin{array}{r}94, \\
6 \\
\end{array}$ & $\begin{array}{r}104 \\
, 0 \\
\end{array}$ & 107,5 & 103,5 & $\begin{array}{r}101 \\
, 4 \\
\end{array}$ & 100,0 & 97,6 & $\begin{array}{r}103 \\
, 2 \\
\end{array}$ & $\begin{array}{r}106 \\
, 8 \\
\end{array}$ \\
\hline Czechia & 84,7 & $\begin{array}{r}88, \\
9 \\
\end{array}$ & $\begin{array}{r}94, \\
5 \\
\end{array}$ & 97,1 & 96,2 & $\begin{array}{r}97, \\
0 \\
\end{array}$ & 100,0 & 99,7 & $\begin{array}{r}103 \\
, 6 \\
\end{array}$ & $\begin{array}{r}107 \\
, 3 \\
\end{array}$ \\
\hline Estonia & 86,6 & $\begin{array}{r}93 \\
7\end{array}$ & $\begin{array}{r}102 \\
, 0\end{array}$ & 104,2 & 103,0 & $\begin{array}{r}101 \\
, 9 \\
\end{array}$ & 100,0 & 99,6 & $\begin{array}{r}103 \\
, 0\end{array}$ & $\begin{array}{r}107 \\
, 3 \\
\end{array}$ \\
\hline Croatia & 89,5 & $\begin{array}{r}94, \\
7\end{array}$ & $\begin{array}{r}103 \\
, 5 \\
\end{array}$ & 106,2 & 102,7 & $\begin{array}{r}101 \\
, 6 \\
\end{array}$ & 100,0 & 98,0 & $\begin{array}{r}102 \\
, 5 \\
\end{array}$ & $\begin{array}{r}105 \\
, 1 \\
\end{array}$ \\
\hline Latvia & 84,9 & $\begin{array}{r}92, \\
6 \\
\end{array}$ & $\begin{array}{r}99 \\
8\end{array}$ & 103,0 & 103,4 & $\begin{array}{r}102 \\
, 2 \\
\end{array}$ & 100,0 & 97,7 & $\begin{array}{r}101 \\
, 6 \\
\end{array}$ & $\begin{array}{r}107 \\
, 5 \\
\end{array}$ \\
\hline $\begin{array}{ll} & \text { Lithuan } \\
\text { ia } & \end{array}$ & 80,7 & $\begin{array}{r}90, \\
6\end{array}$ & $\begin{array}{r}102 \\
, 9\end{array}$ & 108,3 & 107,1 & $\begin{array}{r}104 \\
, 7\end{array}$ & 100,0 & 95,6 & $\begin{array}{r}101 \\
, 4 \\
\end{array}$ & $\begin{array}{r}106 \\
, 9\end{array}$ \\
\hline $\begin{array}{ll} & \text { Hungar } \\
\mathrm{y} & \end{array}$ & 86,8 & $\begin{array}{r}93, \\
2 \\
\end{array}$ & $\begin{array}{r}97, \\
7\end{array}$ & 99,1 & 99,4 & $\begin{array}{r}98, \\
5 \\
\end{array}$ & 100,0 & 99,6 & $\begin{array}{r}104 \\
, 6 \\
\end{array}$ & $\begin{array}{r}107 \\
, 1 \\
\end{array}$ \\
\hline Poland & 85,8 & $\begin{array}{r}91, \\
8\end{array}$ & $\begin{array}{r}96, \\
5\end{array}$ & 98,7 & 98,4 & $\begin{array}{r}98, \\
1\end{array}$ & 100,0 & 98,9 & $\begin{array}{r}103 \\
, 5 \\
\end{array}$ & $\begin{array}{r}106 \\
, 0\end{array}$ \\
\hline $\begin{array}{ll} & \text { Romani } \\
\text { a } & \end{array}$ & 82,6 & $\begin{array}{r}89 \\
4\end{array}$ & $\begin{array}{r}98, \\
0\end{array}$ & 100,4 & 99,2 & $\begin{array}{r}98 \\
7\end{array}$ & 100,0 & 99,3 & $\begin{array}{r}102 \\
, 2\end{array}$ & $\begin{array}{r}108 \\
, 3\end{array}$ \\
\hline $\begin{array}{ll} & \text { Sloveni } \\
\text { a } & \\
\end{array}$ & 91,3 & $\begin{array}{r}95 \\
7\end{array}$ & $\begin{array}{r}101 \\
, 2 \\
\end{array}$ & 103,0 & 101,5 & $\begin{array}{r}100 \\
, 1 \\
\end{array}$ & 100,0 & 98,5 & $\begin{array}{r}102 \\
, 4 \\
\end{array}$ & $\begin{array}{r}105 \\
, 5 \\
\end{array}$ \\
\hline $\begin{array}{lr} & \text { Slovaki } \\
\text { a } & \end{array}$ & 96,3 & $\begin{array}{r}99 \\
1\end{array}$ & $\begin{array}{r}103 \\
, 5 \\
\end{array}$ & 103,7 & 101,9 & $\begin{array}{r}98, \\
0\end{array}$ & 100,0 & 101,8 & $\begin{array}{r}107 \\
, 3 \\
\end{array}$ & $\begin{array}{r}111 \\
, 3 \\
\end{array}$ \\
\hline
\end{tabular}

Source: Eurostat database 2009-2018

In Table 2 we can see the development of the imports of CEE countries during 2009-2018. In Bulgaria, Baltic States and Croatia the decrease of exports was also accompanied by a decrease of imports during 2013-2016. The other analysed CEE countries also faced a small decrease of their imports during 2013-2014 and even in 2016.

Table 2. Import unit value index $(2015=100)$ - total products, all countries of the world

\begin{tabular}{|c|c|c|c|c|c|c|c|c|c|c|}
\hline & $9^{200}$ & $0^{201}$ & $\begin{array}{l}201 \\
1\end{array}$ & $2^{201}$ & $3^{201}$ & $\begin{array}{r}201 \\
4\end{array}$ & $\begin{array}{ll} & 201 \\
5 & \\
\end{array}$ & $6^{201}$ & $\begin{array}{l}201 \\
7\end{array}$ & $8^{201}$ \\
\hline ria & 88,9 & 97,7 & $\begin{array}{r}108 \\
7 \\
\end{array}$ & $\begin{array}{r}112, \\
0 \\
\end{array}$ & $\begin{array}{r}108 \\
5 \\
\end{array}$ & $\begin{array}{r}105 \\
4 \\
\end{array}$ & $\begin{array}{r}100 \\
0\end{array}$ & 96,5 & $\begin{array}{r}103 \\
9 \\
\end{array}$ & $\begin{array}{r}108, \\
7\end{array}$ \\
\hline ia Czech & 83,7 & 89,5 & 95,6 & 99,2 & 97,9 & 97,7 & $\begin{array}{r}100 \\
0\end{array}$ & 98,6 & $\begin{array}{r}104 \\
1\end{array}$ & $\begin{array}{r}107, \\
6\end{array}$ \\
\hline ia $\begin{array}{ll} & \text { Eston } \\
\end{array}$ & 82,8 & 90,2 & 99,4 & $\begin{array}{r}104, \\
0\end{array}$ & $\begin{array}{r}102, \\
3 \\
\end{array}$ & $\begin{array}{r}101 \\
9 \\
\end{array}$ & $\begin{array}{r}100 \\
0\end{array}$ & 97,9 & $\begin{array}{r}102, \\
3\end{array}$ & $\begin{array}{r}106, \\
3\end{array}$ \\
\hline ia & 90,0 & 94,3 & $\begin{array}{r}102, \\
9 \\
\end{array}$ & $\begin{array}{r}107 \\
0 \\
\end{array}$ & $\begin{array}{r}104 \\
4 \\
\end{array}$ & $\begin{array}{r}102, \\
6 \\
\end{array}$ & $\begin{array}{r}100 \\
0 \\
\end{array}$ & 99,1 & $\begin{array}{r}104 \\
7 \\
\end{array}$ & $\begin{array}{r}109, \\
0 \\
\end{array}$ \\
\hline $\begin{array}{ll} & \text { Latvi } \\
\text { a } & \\
\end{array}$ & 86,2 & 91,7 & $\begin{array}{r}100 \\
0 \\
\end{array}$ & $\begin{array}{r}106, \\
2 \\
\end{array}$ & $\begin{array}{r}105 \\
2 \\
\end{array}$ & $\begin{array}{r}103 \\
7 \\
\end{array}$ & $\begin{array}{r}100 \\
0 \\
\end{array}$ & 96,1 & $\begin{array}{r}101 \\
1 \\
\end{array}$ & $\begin{array}{r}105, \\
1 \\
\end{array}$ \\
\hline $\begin{array}{l}\text { Lithu } \\
\text { ania }\end{array}$ & 82,2 & 94,4 & $\begin{array}{r}107 \\
9\end{array}$ & $\begin{array}{r}113 \\
8\end{array}$ & $\begin{array}{r}111 \\
8\end{array}$ & $\begin{array}{r}106, \\
9\end{array}$ & $\begin{array}{r}100 \\
0\end{array}$ & 94,8 & $\begin{array}{r}101, \\
2\end{array}$ & $\begin{array}{r}107, \\
2\end{array}$ \\
\hline $\begin{array}{l}\text { Hung } \\
\text { ary }\end{array}$ & 92,1 & 96,6 & $\begin{array}{r}100 \\
7 \\
\end{array}$ & $\begin{array}{r}102 \\
6 \\
\end{array}$ & $\begin{array}{r}100 \\
7 \\
\end{array}$ & 99,5 & $\begin{array}{r}100 \\
0\end{array}$ & 98,5 & $\begin{array}{r}102, \\
6 \\
\end{array}$ & $\begin{array}{r}105, \\
4\end{array}$ \\
\hline d Polan & 82,5 & 90,3 & 97,3 & $\begin{array}{r}101, \\
2 \\
\end{array}$ & $\begin{array}{r}100 \\
1 \\
\end{array}$ & 99,7 & $\begin{array}{r}100 \\
0 \\
\end{array}$ & 98,6 & $\begin{array}{r}104, \\
8 \\
\end{array}$ & $\begin{array}{r}108, \\
2 \\
\end{array}$ \\
\hline nia $^{\text {Roma }}$ & 84,0 & 90,2 & 98,2 & $\begin{array}{r}101, \\
2 \\
\end{array}$ & $\begin{array}{r}100, \\
6 \\
\end{array}$ & $\begin{array}{r}100, \\
6 \\
\end{array}$ & $\begin{array}{r}100, \\
0\end{array}$ & 99,2 & $\begin{array}{r}104, \\
3 \\
\end{array}$ & $\begin{array}{r}110, \\
7\end{array}$ \\
\hline $\begin{array}{l}\text { Slove } \\
\text { nia }\end{array}$ & 88,4 & 95,8 & $\begin{array}{r}102, \\
9 \\
\end{array}$ & $\begin{array}{r}105 \\
5 \\
\end{array}$ & $\begin{array}{r}103, \\
0 \\
\end{array}$ & $\begin{array}{r}101, \\
1 \\
\end{array}$ & $\begin{array}{r}100 \\
0 \\
\end{array}$ & 98,2 & $\begin{array}{r}103, \\
3 \\
\end{array}$ & $\begin{array}{r}107, \\
4 \\
\end{array}$ \\
\hline kia $^{\text {Slova }}$ & 91,5 & 98,1 & $\begin{array}{r}104, \\
0\end{array}$ & $\begin{array}{r}105 \\
4 \\
\end{array}$ & $\begin{array}{r}103, \\
8 \\
\end{array}$ & 97,4 & $\begin{array}{r}100 \\
0 \\
\end{array}$ & $\begin{array}{r}100 \\
7 \\
\end{array}$ & $\begin{array}{r}104, \\
3 \\
\end{array}$ & $\begin{array}{r}108, \\
3 \\
\end{array}$ \\
\hline
\end{tabular}


In Table 3 we have presented the data for FDI inward in CEE countries (\% of GDP) and we can stress from the dataseries that Slovenia displays the lowest share of FDI inward of GDP (but it is an increasing trend), followed by Lithuania, Poland and Romania. The highest share of FDI inward of GDP can be seen in Estonia, Bulgaria (with a decreasing trend), Czechia, Hungary and Croatia.

Table 3. FDI inward stock share of GDP (2009-2018)

\begin{tabular}{|c|c|c|c|c|c|c|c|c|c|c|}
\hline & $\begin{array}{c}20 \\
09\end{array}$ & $\begin{array}{c}20 \\
10\end{array}$ & $\begin{array}{r}20 \\
11\end{array}$ & $\begin{array}{c}20 \\
12\end{array}$ & $\begin{array}{r}20 \\
13 \\
\end{array}$ & $\begin{array}{r}20 \\
14 \\
\end{array}$ & $\begin{array}{r}20 \\
15 \\
\end{array}$ & $\begin{array}{r}20 \\
16 \\
\end{array}$ & $\begin{array}{r}20 \\
17 \\
\end{array}$ & $\begin{array}{r}20 \\
18 \\
\end{array}$ \\
\hline Bulgaria & $\begin{array}{l}94, \\
8 \\
\end{array}$ & $\begin{array}{l}88, \\
8 \\
\end{array}$ & $\begin{array}{l}79 \\
5 \\
\end{array}$ & $\begin{array}{l}90, \\
2\end{array}$ & $\begin{array}{l}90, \\
5 \\
\end{array}$ & $\begin{array}{l}80, \\
0\end{array}$ & $\begin{array}{l}86, \\
7 \\
\end{array}$ & $\begin{array}{l}80, \\
7\end{array}$ & $\begin{array}{l}87, \\
6 \\
\end{array}$ & $\begin{array}{c}76, \\
0 \\
\end{array}$ \\
\hline Croatia & $\begin{array}{l}52, \\
5\end{array}$ & $\begin{array}{l}52, \\
6\end{array}$ & $\begin{array}{l}45, \\
2\end{array}$ & $\begin{array}{l}52, \\
4\end{array}$ & $\begin{array}{l}51, \\
3\end{array}$ & $\begin{array}{l}50, \\
2\end{array}$ & $\begin{array}{l}52, \\
4\end{array}$ & $\begin{array}{l}53, \\
4\end{array}$ & $\begin{array}{l}60, \\
6\end{array}$ & $\begin{array}{l}54, \\
2\end{array}$ \\
\hline Czechia & $\begin{array}{l}61, \\
0 \\
\end{array}$ & $\begin{array}{l}61, \\
9 \\
\end{array}$ & $\begin{array}{l}52, \\
9 \\
\end{array}$ & $\begin{array}{l}65 \\
8 \\
\end{array}$ & $\begin{array}{l}\text { 64, } \\
0 \\
\end{array}$ & $\begin{array}{l}58, \\
4 \\
\end{array}$ & $\begin{array}{l}62, \\
4 \\
\end{array}$ & $\begin{array}{l}62, \\
4 \\
\end{array}$ & $\begin{array}{l}72, \\
2 \\
\end{array}$ & $\begin{array}{l}64, \\
1 \\
\end{array}$ \\
\hline Estonia & $\begin{array}{l}80, \\
5\end{array}$ & $\begin{array}{l}79 \\
7\end{array}$ & $\begin{array}{l}70, \\
5\end{array}$ & $\begin{array}{l}82, \\
1\end{array}$ & $\begin{array}{l}87, \\
8\end{array}$ & $\begin{array}{l}79, \\
9\end{array}$ & $\begin{array}{l}84, \\
0\end{array}$ & $\begin{array}{l}84, \\
5\end{array}$ & $\begin{array}{l}92, \\
1\end{array}$ & $\begin{array}{l}82, \\
5\end{array}$ \\
\hline Hungary & $\begin{array}{l}75 \\
7\end{array}$ & $\begin{array}{l}69, \\
3\end{array}$ & $\begin{array}{l}60, \\
6\end{array}$ & $\begin{array}{l}81, \\
3\end{array}$ & $\begin{array}{l}80, \\
3\end{array}$ & $\begin{array}{l}71, \\
0\end{array}$ & $\begin{array}{l}68, \\
9\end{array}$ & $\begin{array}{l}64, \\
0\end{array}$ & $\begin{array}{l}64, \\
8\end{array}$ & $\begin{array}{l}57, \\
0\end{array}$ \\
\hline Latvia & $\begin{array}{l}44, \\
4\end{array}$ & $\begin{array}{l}46, \\
0\end{array}$ & $\begin{array}{l}42, \\
5\end{array}$ & $\begin{array}{l}48, \\
1\end{array}$ & $\begin{array}{l}52, \\
7\end{array}$ & $\begin{array}{l}48, \\
1\end{array}$ & $\begin{array}{l}54, \\
6\end{array}$ & $\begin{array}{l}51, \\
4\end{array}$ & $\begin{array}{l}57, \\
5 \\
\end{array}$ & $\begin{array}{l}49, \\
6\end{array}$ \\
\hline Lithuania & $\begin{array}{l}35, \\
4\end{array}$ & $\begin{array}{l}36, \\
1\end{array}$ & $\begin{array}{l}32, \\
7\end{array}$ & $\begin{array}{c}37, \\
2\end{array}$ & $\begin{array}{l}37, \\
8\end{array}$ & $\begin{array}{l}31, \\
8\end{array}$ & $\begin{array}{l}35, \\
4\end{array}$ & $\begin{array}{l}34, \\
1\end{array}$ & $\begin{array}{l}37, \\
3\end{array}$ & $\begin{array}{l}33, \\
3\end{array}$ \\
\hline Poland & $\begin{array}{c}38, \\
0\end{array}$ & $\begin{array}{l}39, \\
1\end{array}$ & $\begin{array}{l}31, \\
1\end{array}$ & $\begin{array}{l}39, \\
7\end{array}$ & $\begin{array}{l}44, \\
2 \\
\end{array}$ & $\begin{array}{l}38, \\
7\end{array}$ & $\begin{array}{l}38, \\
9\end{array}$ & $\begin{array}{l}40, \\
0\end{array}$ & $\begin{array}{l}45, \\
3 \\
\end{array}$ & $\begin{array}{l}39, \\
5\end{array}$ \\
\hline Romania & $\begin{array}{l}40 \\
7\end{array}$ & $2_{2}^{41,}$ & $\begin{array}{l}37 \\
7\end{array}$ & $\begin{array}{l}44, \\
4\end{array}$ & $\begin{array}{l}43, \\
1\end{array}$ & $\begin{array}{l}36, \\
6\end{array}$ & $\begin{array}{l}39 \\
4\end{array}$ & $\begin{array}{l}39, \\
3\end{array}$ & $\begin{array}{l}42, \\
9\end{array}$ & $\begin{array}{c}39, \\
1 \\
\end{array}$ \\
\hline Slovakia & $\begin{array}{l}59, \\
0\end{array}$ & $\begin{array}{c}56, \\
2\end{array}$ & $\begin{array}{l}52, \\
9\end{array}$ & $\begin{array}{l}59, \\
0\end{array}$ & $\begin{array}{l}58, \\
9\end{array}$ & $\begin{array}{l}49, \\
2\end{array}$ & $\begin{array}{l}52, \\
4\end{array}$ & $\begin{array}{l}52, \\
9\end{array}$ & $\begin{array}{l}58, \\
4\end{array}$ & $\begin{array}{l}53, \\
6\end{array}$ \\
\hline Slovenia & $\begin{array}{l}22, \\
4\end{array}$ & $\begin{array}{l}22, \\
2\end{array}$ & $\begin{array}{l}22, \\
4\end{array}$ & $\begin{array}{l}26, \\
3\end{array}$ & $\begin{array}{l}25, \\
5\end{array}$ & $\begin{array}{l}24, \\
8\end{array}$ & $\begin{array}{l}29, \\
3\end{array}$ & $\begin{array}{l}30 \\
6\end{array}$ & $\begin{array}{l}33, \\
8\end{array}$ & $\begin{array}{l}31, \\
0\end{array}$ \\
\hline
\end{tabular}

Source: UNCTAD database

Although it displays the lowest share of FDI inward stock of GDP, Slovenia display a high share of exports of GDP and a high share of imports of GDP, together with Slovakia and Hungary. Poland and Romania display low shares of exports and imports of GDP and low share of FDI inward stock of GDP, while Croatia display large FDI inflows and stock, but a low share of exports of GDP and a low share of imports of GDP in the CEE region (Tables 4 and 5).

Table 4. Total exports share of GDP (\%)

\begin{tabular}{|c|c|c|c|c|c|c|c|c|c|c|}
\hline & $\begin{array}{l}200 \\
9\end{array}$ & 2010 & $\begin{array}{r}20 \\
11\end{array}$ & $\begin{array}{c}20 \\
12\end{array}$ & $\begin{array}{c}20 \\
13\end{array}$ & $\begin{array}{l}20 \\
14\end{array}$ & 2015 & $\begin{array}{c}20 \\
16\end{array}$ & $\begin{array}{c}20 \\
17\end{array}$ & 2018 \\
\hline $\begin{array}{l}\text { Bulgari } \\
\mathbf{a}\end{array}$ & 42,3 & 50,1 & $\begin{array}{l}59, \\
0\end{array}$ & $\begin{array}{l}60, \\
8\end{array}$ & $\begin{array}{l}64, \\
8\end{array}$ & $\begin{array}{l}64, \\
9\end{array}$ & 64,5 & $\begin{array}{l}64, \\
7\end{array}$ & $\begin{array}{l}68, \\
1\end{array}$ & 66,9 \\
\hline Croatia & 32,6 & 36,1 & $\begin{array}{l}38, \\
8 \\
\end{array}$ & $\begin{array}{l}39, \\
5\end{array}$ & $\begin{array}{l}40, \\
4\end{array}$ & $\begin{array}{l}43, \\
3 \\
\end{array}$ & 46,3 & $\begin{array}{l}47, \\
6\end{array}$ & $\begin{array}{l}50, \\
0\end{array}$ & 50,5 \\
\hline Czechia & 58,6 & 66,0 & $\begin{array}{l}71, \\
3\end{array}$ & $\begin{array}{l}76, \\
1\end{array}$ & $\begin{array}{l}76, \\
8\end{array}$ & $\begin{array}{l}82, \\
5\end{array}$ & 81,0 & $\begin{array}{l}79, \\
5\end{array}$ & $\begin{array}{l}79, \\
7\end{array}$ & 78,3 \\
\hline Estonia & 60,5 & 74,4 & $\begin{array}{l}85, \\
8\end{array}$ & $\begin{array}{l}85, \\
5\end{array}$ & $\begin{array}{l}84, \\
0\end{array}$ & $\begin{array}{l}81, \\
3\end{array}$ & 76,8 & ${ }_{4}^{77,}$ & ${ }_{1}^{76,}$ & 74,2 \\
\hline $\begin{array}{l}\text { Hungar } \\
\mathbf{y}\end{array}$ & 74,4 & 81,7 & $\begin{array}{l}86, \\
5\end{array}$ & $\begin{array}{l}86, \\
3\end{array}$ & $\begin{array}{l}85, \\
5\end{array}$ & $\begin{array}{l}87, \\
4\end{array}$ & 87,9 & $\begin{array}{l}87, \\
1\end{array}$ & $\begin{array}{l}87, \\
1\end{array}$ & 84,9 \\
\hline Latvia & 42,4 & 53,5 & $\begin{array}{l}57, \\
7\end{array}$ & ${ }_{2}^{61,}$ & $\begin{array}{l}60, \\
2\end{array}$ & $\begin{array}{l}61, \\
2\end{array}$ & 60,7 & $\begin{array}{l}60, \\
4\end{array}$ & $\begin{array}{l}62, \\
1\end{array}$ & 61,3 \\
\hline $\begin{array}{l}\text { Lithuan } \\
\text { ia }\end{array}$ & 51,9 & 64,1 & $\begin{array}{l}73, \\
0\end{array}$ & $\begin{array}{l}78, \\
4\end{array}$ & $\begin{array}{l}78, \\
9\end{array}$ & $\begin{array}{l}72, \\
3\end{array}$ & 68,8 & $\begin{array}{l}67, \\
5\end{array}$ & $\begin{array}{l}73, \\
6\end{array}$ & 75,6 \\
\hline Poland & 37,1 & 40,0 & $\begin{array}{l}42, \\
5\end{array}$ & $\begin{array}{l}44, \\
4\end{array}$ & $\begin{array}{l}46, \\
3\end{array}$ & $\begin{array}{l}47, \\
5\end{array}$ & 49,5 & $\begin{array}{l}52, \\
2\end{array}$ & $\begin{array}{l}54, \\
3\end{array}$ & 55,6 \\
\hline $\begin{array}{l}\text { Romani } \\
\text { a }\end{array}$ & 26,0 & 32,4 & & $\begin{array}{l}37, \\
4\end{array}$ & $\begin{array}{l}39, \\
8\end{array}$ & $\begin{array}{l}{ }_{1}^{41,} \\
1\end{array}$ & 41,0 & $\begin{array}{l}41, \\
1\end{array}$ & $\begin{array}{l}41, \\
4\end{array}$ & 41,6 \\
\hline $\begin{array}{l}\text { Slovaki } \\
\text { a }\end{array}$ & 68,0 & 77,3 & & $\begin{array}{l}91, \\
0\end{array}$ & $\begin{array}{l}93, \\
6\end{array}$ & $\begin{array}{l}91, \\
5\end{array}$ & 92,0 & $\begin{array}{l}93, \\
7\end{array}$ & $\begin{array}{l}95, \\
1\end{array}$ & 96,1 \\
\hline $\begin{array}{l}\text { Sloveni } \\
\text { a }\end{array}$ & 57,2 & 64,2 & ${ }_{2}^{70,}$ & $\begin{array}{l}72, \\
9\end{array}$ & ${ }_{2}^{74,}$ & $\begin{array}{l}76, \\
1\end{array}$ & 77,1 & $\begin{array}{l}77, \\
9\end{array}$ & $\begin{array}{l}83, \\
1\end{array}$ & 85,3 \\
\hline
\end{tabular}


Table 5. Total imports share of GDP (\%)

\begin{tabular}{|c|c|c|c|c|c|c|c|c|c|c|}
\hline & $\begin{array}{l}200 \\
9\end{array}$ & $\begin{array}{l}201 \\
0\end{array}$ & $\begin{array}{l}20 \\
11\end{array}$ & $\begin{array}{l}201 \\
2\end{array}$ & $\begin{array}{l}201 \\
3\end{array}$ & $\begin{array}{l}20 \\
14\end{array}$ & $\begin{array}{l}201 \\
5\end{array}$ & $\begin{array}{r}20 \\
16\end{array}$ & $\begin{array}{l}201 \\
7\end{array}$ & $\begin{array}{c}20 \\
18\end{array}$ \\
\hline Bulgaria & 50,6 & 53,0 & $\begin{array}{l}58, \\
7 \\
\end{array}$ & 64,0 & 65,3 & $\begin{array}{l}65, \\
8 \\
\end{array}$ & 63,5 & $\begin{array}{l}59 \\
7 \\
\end{array}$ & 63,7 & $\begin{array}{l}64, \\
3 \\
\end{array}$ \\
\hline Croatia & 38,2 & 38,0 & $\begin{array}{l}40, \\
8\end{array}$ & 41,2 & 42,5 & $\begin{array}{l}43, \\
6\end{array}$ & 46,1 & $\begin{array}{l}46, \\
5\end{array}$ & 49,4 & $\begin{array}{l}51, \\
3 \\
\end{array}$ \\
\hline Czechia & 54,8 & 62,9 & $\begin{array}{l}67, \\
4\end{array}$ & 71,3 & 71,1 & $\begin{array}{l}76, \\
1\end{array}$ & 75,0 & $\begin{array}{l}71, \\
8\end{array}$ & 72,2 & $\begin{array}{l}72, \\
0\end{array}$ \\
\hline Estonia & 55,5 & 68,0 & $\begin{array}{l}80 \text {, } \\
0\end{array}$ & 83,9 & 81,3 & $\begin{array}{l}77, \\
8\end{array}$ & 72,9 & $\begin{array}{l}73, \\
5\end{array}$ & 71,8 & $\begin{array}{l}70 \text {, } \\
7\end{array}$ \\
\hline Hungary & 70,3 & 76,4 & $\begin{array}{l}80, \\
4\end{array}$ & 79,5 & 78,6 & $\begin{array}{l}81, \\
0\end{array}$ & 79,9 & $\begin{array}{l}78, \\
4\end{array}$ & 79,8 & $\begin{array}{l}80 \text {, } \\
5\end{array}$ \\
\hline Latvia & 44,0 & 55,0 & $\begin{array}{l}62, \\
7\end{array}$ & 65,6 & 63,8 & $\begin{array}{l}\text { 63, } \\
2\end{array}$ & 61,6 & $\begin{array}{l}59, \\
4\end{array}$ & 61,9 & $\begin{array}{l}61, \\
4 \\
\end{array}$ \\
\hline Lithuania & 53,6 & 66,1 & $\begin{array}{l}75, \\
7\end{array}$ & 77,8 & 77,3 & $\begin{array}{l}70, \\
5\end{array}$ & 69,8 & $\begin{array}{l}66, \\
8\end{array}$ & 71,2 & $\begin{array}{l}73, \\
6\end{array}$ \\
\hline Poland & 38,0 & 42,0 & $\begin{array}{l}44, \\
5\end{array}$ & 44,8 & 44,3 & $\begin{array}{l}46, \\
1\end{array}$ & 46,4 & $\begin{array}{l}48, \\
1\end{array}$ & 50,1 & $\begin{array}{l}52, \\
1 \\
\end{array}$ \\
\hline Romania & 32,4 & 38,8 & $\begin{array}{l}42, \\
8\end{array}$ & 42,5 & 40,6 & $\begin{array}{l}41, \\
6\end{array}$ & 41,6 & $\begin{array}{l}42, \\
1\end{array}$ & 43,6 & $\begin{array}{l}44, \\
8 \\
\end{array}$ \\
\hline Slovakia & 68,2 & 77,6 & $\begin{array}{l}84, \\
3\end{array}$ & 85,5 & 88,0 & $\begin{array}{l}86, \\
7\end{array}$ & 88,9 & $\begin{array}{l}90, \\
7\end{array}$ & 92,9 & $\begin{array}{l}94, \\
0\end{array}$ \\
\hline Slovenia & 55,8 & 63,2 & $\begin{array}{l}69, \\
\mathrm{O}\end{array}$ & 69,5 & 69,5 & $\begin{array}{l}69, \\
4\end{array}$ & 69,1 & $\begin{array}{l}69, \\
4\end{array}$ & 74,3 & $\begin{array}{l}77, \\
0\end{array}$ \\
\hline
\end{tabular}

Source: World Bank database

Large deficits of the foreign trade with goods can be seen in Croatia, Latvia and Romania. Bulgaria faced large commercial deficits until 2013, but after then, it faced a decreasing trend of its commercial deficit. Czechia and Hungary display a high surplus of their commercial balance for goods and, during the last analysed years, we can see surplus of the trade balance for goods also in Slovenia and Slovakia (Slovakia displays a descending trend of its commercial surplus). Poland also reduced its commercial deficit and even faced three years of surplus during 20152017 (Table 6).

Table 6. Trade balance (goods) share of GDP

\begin{tabular}{|c|c|c|c|c|c|c|c|c|c|c|}
\hline & $9 \begin{array}{l}200 \\
9\end{array}$ & $\begin{array}{l}201 \\
0\end{array}$ & $\begin{array}{l}201 \\
1\end{array}$ & 201 & $\begin{array}{ll} & 201 \\
3 & \\
\end{array}$ & $\begin{array}{r}201 \\
4 \quad \\
\end{array}$ & $\begin{array}{ll} & 201 \\
5 & \\
\end{array}$ & $\begin{array}{ll} & 201 \\
& \\
\end{array}$ & $\begin{array}{r}201 \\
7\end{array}$ & $\begin{array}{ll} & 201 \\
8 & \\
\end{array}$ \\
\hline $\begin{array}{ll} & \text { Bulgari } \\
\text { a } & \\
\end{array}$ & $\begin{array}{r}- \\
11,13 \\
\end{array}$ & $\begin{array}{r}- \\
9,37 \\
\end{array}$ & $\begin{array}{r}- \\
6,51 \\
\end{array}$ & 9,51 & $\begin{array}{r}- \\
7,00 \\
\end{array}$ & $\begin{array}{r}- \\
6,48 \\
\end{array}$ & $\begin{array}{r}- \\
5,79 \\
\end{array}$ & $\begin{array}{r}- \\
2,04 \\
\end{array}$ & $\begin{array}{r}- \\
1,48 \\
\end{array}$ & $\begin{array}{r}- \\
4,12 \\
\end{array}$ \\
\hline Croatia & $\begin{array}{r}- \\
16,61\end{array}$ & $\begin{array}{r}- \\
13,15 \\
\end{array}$ & $\begin{array}{r}- \\
14,41 \\
\end{array}$ & $\begin{array}{r}- \\
14,74 \\
\end{array}$ & $\begin{array}{r}- \\
15,25 \\
\end{array}$ & $\begin{array}{r}- \\
14,93 \\
\end{array}$ & $\begin{array}{r}- \\
15,12 \\
\end{array}$ & $\begin{array}{r}- \\
15,83 \\
\end{array}$ & $\begin{array}{r}- \\
15,77 \\
\end{array}$ & $\begin{array}{r}- \\
18,18 \\
\end{array}$ \\
\hline $\begin{array}{ll} & \text { Czechi } \\
\text { a } & \\
\end{array}$ & 1,64 & 0,99 & 1,87 & 3,04 & 4,08 & 5,10 & $\begin{array}{r}4,07 \\
9 \\
\end{array}$ & 5,15 & 5,10 & 4,14 \\
\hline Estonia & $\begin{array}{r}- \\
5,12 \\
\end{array}$ & $\begin{array}{r}- \\
2,75 \\
\end{array}$ & $\begin{array}{r}- \\
2,10 \\
\end{array}$ & $\begin{array}{r}- \\
7,13 \\
\end{array}$ & $\begin{array}{r}- \\
4,88 \\
\end{array}$ & $\begin{array}{r}- \\
5,02 \\
\end{array}$ & $\begin{array}{r}- \\
4,33 \\
\end{array}$ & $\begin{array}{r}- \\
3,60 \\
\end{array}$ & $\begin{array}{r}- \\
3,54 \\
\end{array}$ & $\begin{array}{r}- \\
3,88 \\
\end{array}$ \\
\hline Hungar & 2,81 & 2,64 & 2,89 & 2,93 & 3,27 & 2,01 & 3,65 & 4,02 & 1,54 & $\begin{array}{r}- \\
1,05 \\
\end{array}$ \\
\hline Latvia & $\begin{array}{r}- \\
8,38 \\
\end{array}$ & $\begin{array}{r}- \\
8,59 \\
\end{array}$ & $\begin{array}{r}- \\
12,26 \\
\end{array}$ & $\begin{array}{r}- \\
12,04 \\
\end{array}$ & $\begin{array}{r}- \\
11,49 \\
\end{array}$ & $\begin{array}{r}- \\
10,06\end{array}$ & $\begin{array}{r}- \\
9,03 \\
\end{array}$ & $\begin{array}{r}- \\
7,52 \\
\end{array}$ & $\begin{array}{r}- \\
8,53 \\
\end{array}$ & $\begin{array}{r}- \\
8,72 \\
\end{array}$ \\
\hline $\begin{array}{l}\text { Lithua } \\
\text { nia }\end{array}$ & $\begin{array}{r}- \\
4,33 \\
\end{array}$ & $\begin{array}{r}- \\
5,87 \\
\end{array}$ & $\begin{array}{r}- \\
6,59 \\
\end{array}$ & $\begin{array}{r}- \\
3,301 \\
\end{array}$ & $\begin{array}{r}- \\
2,63 \\
\end{array}$ & $\begin{array}{r}- \\
2,55 \\
\end{array}$ & $\begin{array}{r}- \\
5,30 \\
\end{array}$ & $\begin{array}{r}- \\
4,55 \\
\end{array}$ & $\begin{array}{r}- \\
4,62 \\
\end{array}$ & $\begin{array}{r}- \\
5,86 \\
\end{array}$ \\
\hline Poland & $\begin{array}{r}- \\
2,43 \\
\end{array}$ & $\begin{array}{r}- \\
3,02 \\
\end{array}$ & $\begin{array}{r}- \\
3,49 \\
\end{array}$ & $\begin{array}{r}- \\
2,08 \\
\end{array}$ & $\begin{array}{r}- \\
0,08 \\
\end{array}$ & $\begin{array}{r}- \\
0,79 \\
\end{array}$ & 0,51 & 0,68 & 0,30 & $\begin{array}{r}- \\
1,04 \\
\end{array}$ \\
\hline $\begin{array}{lll} & & \text { Romani } \\
\text { a } & \\
\end{array}$ & $\begin{array}{r}- \\
7,26 \\
\end{array}$ & $\begin{array}{r}- \\
7,63 \\
\end{array}$ & $\begin{array}{r}- \\
7,06 \\
\end{array}$ & $\begin{array}{r}- \\
6,93 \\
\end{array}$ & $\begin{array}{r}- \\
4,03 \\
\end{array}$ & $\begin{array}{r}- \\
4,34 \\
\end{array}$ & $\begin{array}{r}- \\
4,85 \\
\end{array}$ & $\begin{array}{r}- \\
5,47 \\
\end{array}$ & $\begin{array}{r}- \\
6,48 \\
\end{array}$ & $\begin{array}{r}- \\
7,28 \\
\end{array}$ \\
\hline $\begin{array}{ll} & \text { Slovaki } \\
\text { a } & \\
\end{array}$ & 0,36 & 0,11 & $\begin{array}{r}- \\
0,053 \\
\end{array}$ & 3,44 & 3,92 & 3,62 & 1,32 & 2,01 & 0,81 & 0,05 \\
\hline $\begin{array}{ll} & \text { Sloveni } \\
\text { a } & \\
\end{array}$ & $\begin{array}{r}- \\
1,16 \\
\end{array}$ & $\begin{array}{r}- \\
2,06\end{array}$ & $\begin{array}{r}- \\
2,63 \\
\end{array}$ & $\begin{array}{r}- \\
0,22 \\
\end{array}$ & 1,95 & 3,14 & 3,79 & 3,80 & 3,63 & 2,50 \\
\hline
\end{tabular}

Hungary, Slovenia and Slovakia display the highest share of FDI stock in the manufacturing sectors. That is why, Slovenia display a high share of its exports of GDP, although the share of FDI inward stock of GDP is the lowest in the entire CEE region. Hungary displays a high share of FDI inward stock of GDP as well as the share of FDI stock 
in the manufacturing sectors and that boosts its exports and generally its foreign trade with goods. Slovakia also displays a high share of its exports of GDP, due to its important share of FDI stock in the manufacturing sectors. Latvia, Estonia, Bulgaria and Lithuania display the lowest share of FDI inward stock in the manufacturing sectors. This low share explains the low share of the exports of GDP of those countries (Table 7).

Table 7. FDI stock in manufacturing sectors in Romania, Bulgaria (\% of total FDI inward stock)

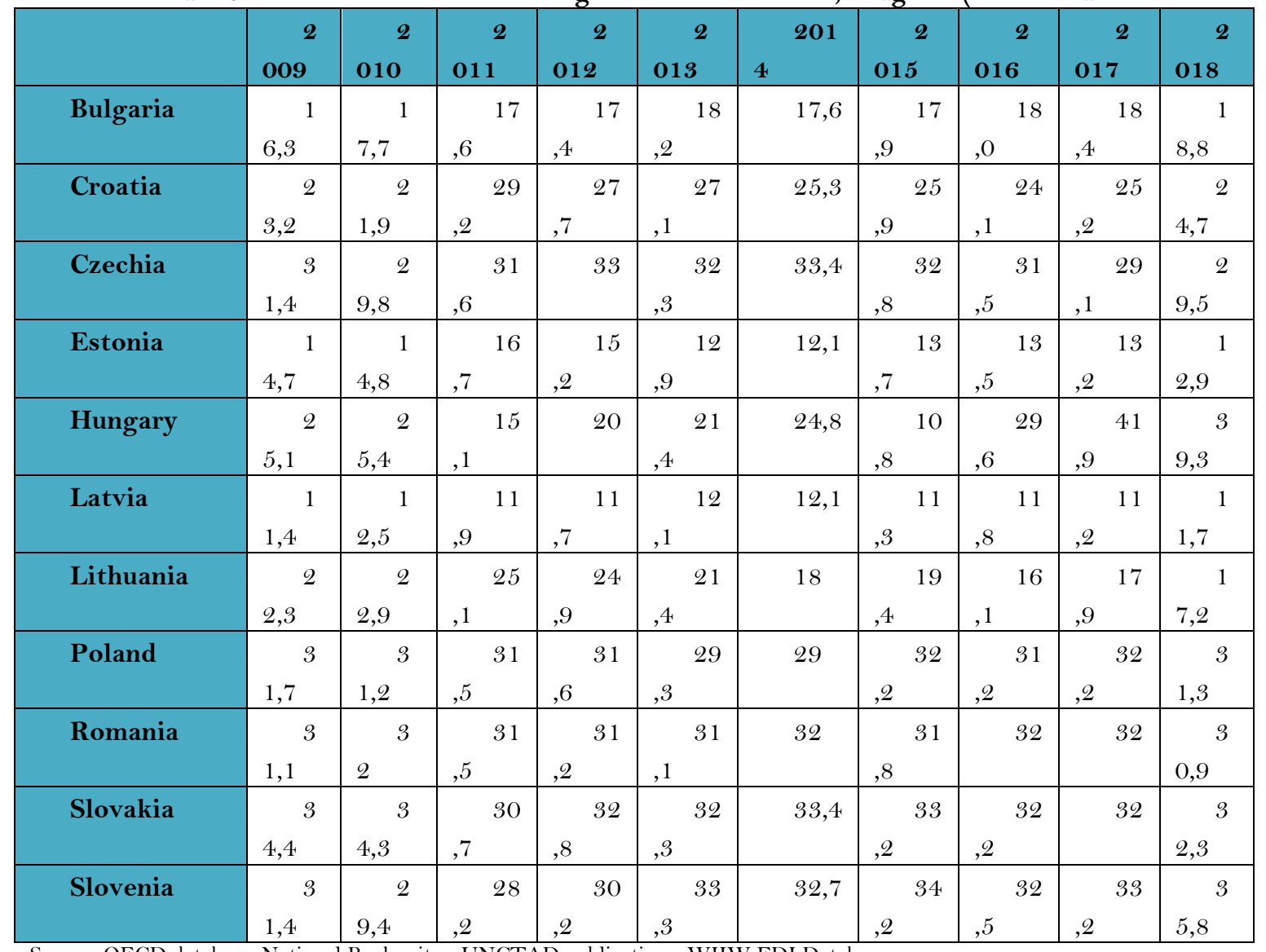

Source: OECD database, National Banks sites, UNCTAD publications, WIIW FDI Database

High shares of FDI stock of GDP and high shares of FDI stock in the manufactured sectors are associated with high share of exports of manufactured goods of total exports in Czechia and Hungary that also display commercial surplus, but also in Slovakia where the commercial surplus is lower than in Czechia and in Hungary. Although Bulgaria display a high share of FDI stock of GDP, the share of FDI stock in manufacturing sectors is low and the exports of manufactured good is also low, so they are positively correlated. In Croatia, Estonia, Latvia and Lithuania we can also see low shares of FDI stocks in the manufacturing sectors and a low share of manufactured goods, too (Table 8). These last 4 countries display the highest commercial deficits in the CEE region (Table 6).

Table 8. Exports of manufactured goods (\% of total exports of goods)

\begin{tabular}{|c|c|c|c|c|c|c|c|c|c|c|}
\hline & $09^{20}$ & $10^{20}$ & $1^{201}$ & $2^{201}$ & $3^{201}$ & $\begin{array}{r}201 \\
4 \quad\end{array}$ & $5^{201}$ & $16^{20}$ & $\begin{array}{ll} & 201 \\
7 & \end{array}$ & $8^{201}$ \\
\hline Bulgaria & $\begin{array}{r}53, \\
1 \\
\end{array}$ & 54 & 51,3 & 50,2 & 50,3 & 54,7 & 57,8 & $\begin{array}{r}59, \\
2 \\
\end{array}$ & 57,2 & 58,9 \\
\hline Croatia & $\begin{array}{r}76 \\
7 \\
\end{array}$ & $\begin{array}{r}75 \\
4 \\
\end{array}$ & 74 & 71 & 68,4 & 68,6 & 72,5 & $\begin{array}{r}80, \\
1 \\
\end{array}$ & 71,3 & 79,7 \\
\hline Czechia & $\begin{array}{r}97, \\
3\end{array}$ & $\begin{array}{r}99, \\
2 \\
\end{array}$ & $\begin{array}{r}104, \\
1 \\
\end{array}$ & $\begin{array}{r}102, \\
1 \\
\end{array}$ & $\begin{array}{r}103, \\
9 \\
\end{array}$ & $\begin{array}{r}105, \\
6 \\
\end{array}$ & $\begin{array}{r}108 \\
9 \\
\end{array}$ & 112 & $\begin{array}{r}113 \\
4 \\
\end{array}$ & $\begin{array}{r}113, \\
9 \\
\end{array}$ \\
\hline Estonia & $\begin{array}{r}88, \\
1 \\
\end{array}$ & $\begin{array}{r}80, \\
3 \\
\end{array}$ & 79,3 & 83 & 84,5 & 78,2 & 76,2 & $\begin{array}{r}75, \\
8 \\
\end{array}$ & 74,7 & 75,1 \\
\hline Hungary & $\begin{array}{r}85 \\
8 \\
\end{array}$ & $\begin{array}{r}89, \\
2 \\
\end{array}$ & 90,9 & 92,1 & 95 & 96 & 98,6 & 100 & $\begin{array}{r}101, \\
5\end{array}$ & $\begin{array}{r}101, \\
6 \\
\end{array}$ \\
\hline Latvia & $\begin{array}{r}61, \\
6 \\
\end{array}$ & $\begin{array}{r}57 \\
8 \\
\end{array}$ & 58 & 54,9 & 53,8 & 54,5 & 59 & $\begin{array}{r}60 \\
1 \\
\end{array}$ & 60,9 & 63,5 \\
\hline $\begin{array}{ll} & \text { Lithuani } \\
\text { a } & \\
\end{array}$ & 59 & 57 & 55,2 & 53,8 & 54,3 & 59,6 & 60,7 & $\begin{array}{r}63 \\
7 \\
\end{array}$ & 63,5 & 63,8 \\
\hline Poland & 82 & $\begin{array}{r}79 \\
4\end{array}$ & 79,6 & 75,8 & 78,9 & 79 & 80,2 & $\begin{array}{r}80 \\
3\end{array}$ & 77,6 & 82,4 \\
\hline
\end{tabular}




\begin{tabular}{|r|r|r|r|r|r|r|r|r|r|}
\hline Romania & 95, & 89, & & & & & & & \\
& 9 & 4 & 87,4 & 87,1 & 85,3 & 85,5 & 86,5 & 87,8 & 90,2 \\
\hline Slovakia & 90, & 89, & & & & & 93, & \\
\hline \\
Slovenia
\end{tabular}

Source: authors' calculations based on data from UNCTAD database

Imports of manufactured goods are high in Czechia, Hungary, Slovakia, but also in Romania where the FDI inward stock in manufactured sectors is low. In Romania there are high commercial deficits because of high imports of manufactured goods. In Bulgaria and Lithuania, the share of the imports of manufactured goods are the lowest in the CEE region, but the share of manufactured exports is also low because of a low share of FDI inward stock in the manufacturing economic sectors (Table 9).

Table 9. Imports of manufactured goods (\% of total imports of goods)

\begin{tabular}{|c|c|c|c|c|c|c|c|c|c|c|}
\hline & $09^{20}$ & $10^{20}$ & $11^{20}$ & $12^{20}$ & $13^{20}$ & $14^{20}$ & $15^{20}$ & $16^{20}$ & $\begin{array}{r}201 \\
7\end{array}$ & $\begin{array}{ll} & 201 \\
8 & \\
\end{array}$ \\
\hline Bulgaria & $\begin{array}{r}61 \\
7\end{array}$ & $\begin{array}{r}59 \\
1\end{array}$ & $\begin{array}{r}57 \\
3\end{array}$ & $\begin{array}{r}57 \\
2\end{array}$ & $\begin{array}{r}57 \\
8\end{array}$ & $\begin{array}{r}63, \\
2\end{array}$ & $\begin{array}{r}64 \\
5\end{array}$ & $\begin{array}{r}68 \\
6\end{array}$ & 65,4 & 66,1 \\
\hline Croatia & $\begin{array}{r}75 \\
7 \\
\end{array}$ & $\begin{array}{r}72, \\
8 \\
\end{array}$ & $\begin{array}{r}68, \\
4 \\
\end{array}$ & $\begin{array}{r}66, \\
6 \\
\end{array}$ & $\begin{array}{r}66, \\
4 \\
\end{array}$ & $\begin{array}{r}69 \\
2 \\
\end{array}$ & $\begin{array}{r}72 \\
7 \\
\end{array}$ & $\begin{array}{r}78, \\
6 \\
\end{array}$ & 72,5 & 77,5 \\
\hline Czechia & $\begin{array}{r}84 \\
4 \\
\end{array}$ & $\begin{array}{r}85 \\
5 \\
\end{array}$ & 89 & $\begin{array}{r}85 \\
5 \\
\end{array}$ & $\begin{array}{r}87 \\
1 \\
\end{array}$ & $\begin{array}{r}90 \\
9 \\
\end{array}$ & $\begin{array}{r}96 \\
4 \\
\end{array}$ & $\begin{array}{r}99 \\
8 \\
\end{array}$ & $\begin{array}{r}101 \\
8 \\
\end{array}$ & $\begin{array}{r}102, \\
5\end{array}$ \\
\hline Estonia & $\begin{array}{r}81, \\
3\end{array}$ & $\begin{array}{r}81, \\
1\end{array}$ & $\begin{array}{r}79 \\
8\end{array}$ & $\begin{array}{r}79 \\
7\end{array}$ & $\begin{array}{r}80 \\
4\end{array}$ & $\begin{array}{r}79 \\
8\end{array}$ & $\begin{array}{r}80 \\
5\end{array}$ & 80 & 82,1 & 77,6 \\
\hline Hungary & $\begin{array}{r}73 \\
9 \\
\end{array}$ & $\begin{array}{r}74 \\
9 \\
\end{array}$ & $\begin{array}{r}75 \\
4 \\
\end{array}$ & $\begin{array}{r}77 \\
5 \\
\end{array}$ & $\begin{array}{r}80 \\
8 \\
\end{array}$ & $\begin{array}{r}80 \\
7 \\
\end{array}$ & $\begin{array}{r}85 \\
4 \\
\end{array}$ & $\begin{array}{r}86, \\
7 \\
\end{array}$ & 88,9 & 88 \\
\hline Latvia & $\begin{array}{r}57 \\
6 \\
\end{array}$ & $\begin{array}{r}60 \\
6 \\
\end{array}$ & $\begin{array}{r}60 \\
6 \\
\end{array}$ & $\begin{array}{r}56, \\
8 \\
\end{array}$ & $\begin{array}{r}57, \\
2 \\
\end{array}$ & $\begin{array}{r}60 \\
5 \\
\end{array}$ & 65 & $\begin{array}{r}66, \\
5 \\
\end{array}$ & 67,8 & 70,9 \\
\hline Lithuania & 57 & $\begin{array}{r}53, \\
2 \\
\end{array}$ & $\begin{array}{r}52 \\
7 \\
\end{array}$ & $\begin{array}{r}50 \\
7 \\
\end{array}$ & $\begin{array}{r}53, \\
1 \\
\end{array}$ & $\begin{array}{r}60 \\
1 \\
\end{array}$ & $\begin{array}{r}61 \\
7 \\
\end{array}$ & $\begin{array}{r}65, \\
6 \\
\end{array}$ & 63,8 & 62,7 \\
\hline Poland & $\begin{array}{r}77 \\
6 \\
\end{array}$ & $\begin{array}{r}75 \\
5 \\
\end{array}$ & $\begin{array}{r}73 \\
7 \\
\end{array}$ & $\begin{array}{r}69, \\
6 \\
\end{array}$ & $\begin{array}{r}74 \\
6 \\
\end{array}$ & $\begin{array}{r}74 \\
4 \\
\end{array}$ & $\begin{array}{r}77 \\
8 \\
\end{array}$ & $\begin{array}{r}76 \\
7 \\
\end{array}$ & 74,4 & 78,4 \\
\hline Romania & $\begin{array}{r}88, \\
7 \\
\end{array}$ & $\begin{array}{r}83, \\
2 \\
\end{array}$ & $\begin{array}{r}82, \\
4 \\
\end{array}$ & $\begin{array}{r}80 \\
8 \\
\end{array}$ & $\begin{array}{r}82, \\
5 \\
\end{array}$ & 82 & 85 & $\begin{array}{r}84, \\
4 \\
\end{array}$ & 83,1 & 85,4 \\
\hline Slovakia & $\begin{array}{r}80 \\
7\end{array}$ & $\begin{array}{r}78 \\
7\end{array}$ & 74 & $\begin{array}{r}77 \\
3\end{array}$ & $\begin{array}{r}77 \\
8\end{array}$ & $\begin{array}{r}80 \\
8\end{array}$ & $\begin{array}{r}84 \\
9\end{array}$ & $\begin{array}{r}86, \\
6\end{array}$ & 84,5 & 84,3 \\
\hline Slovenia & $\begin{array}{r}74, \\
3\end{array}$ & $\begin{array}{r}71 \\
6\end{array}$ & $\begin{array}{r}68, \\
2\end{array}$ & $\begin{array}{r}67 \\
7\end{array}$ & $\begin{array}{r}70 \\
7\end{array}$ & $\begin{array}{r}72, \\
1\end{array}$ & $\begin{array}{r}73 \\
7\end{array}$ & $\begin{array}{r}76, \\
6\end{array}$ & 76 & 76 \\
\hline
\end{tabular}

Source: authors' calculations based on data from UNCTAD database

Czechia, Slovenia and Slovakia display large commercial surplus both for total commercial balance of goods, but also for the commercial balance of the manufactured goods. Hungary and Poland display surplus for the trade of the manufactured goods, but they faced deficits for the trade of total goods in 2018, although in the previous years, all 5 countries faced surplus both for the balance of the manufactured goods, but also for the commercial balance of total goods. Romania, Lithuania and Croatia display commercial deficits both for the entire commercial balance of goods and for the balance of the manufactured goods. Latvia and Bulgaria display large deficits for its commercial balance of manufactured goods, but also for the commercial balance of total goods (Table 10).

Table 10. Trade balance in manufactured sectors (\% of total trade balance in goods)

\begin{tabular}{|c|c|c|c|c|c|c|c|c|c|c|}
\hline & $\begin{array}{l}200 \\
9 \\
\end{array}$ & $\begin{array}{l}201 \\
0 \\
\end{array}$ & $\begin{array}{r}20 \\
11 \\
\end{array}$ & $\begin{array}{l}201 \\
2 \\
\end{array}$ & $\begin{array}{l}201 \\
3 \\
\end{array}$ & $\begin{array}{r}20 \\
14 \\
\end{array}$ & $\begin{array}{r}2 \\
015 \\
\end{array}$ & $\begin{array}{r}2 \\
016 \\
\end{array}$ & $\begin{array}{r}2 \\
017 \\
\end{array}$ & $\begin{array}{l}201 \\
8 \\
\end{array}$ \\
\hline $\begin{array}{l}\text { Bulga } \\
\text { ria }\end{array}$ & $\begin{array}{l}85 \\
9 \\
\end{array}$ & $\begin{array}{l}79, \\
6 \\
\end{array}$ & $\begin{array}{l}99, \\
8 \\
\end{array}$ & $\begin{array}{l}91, \\
8 \\
\end{array}$ & $\begin{array}{l}112 \\
, 5 \\
\end{array}$ & $\begin{array}{c}12 \\
7,9 \\
\end{array}$ & $\begin{array}{r}1 \\
20,3 \\
\end{array}$ & $90^{2}$ & $\begin{array}{r}3 \\
57,3 \\
\end{array}$ & 154 \\
\hline $\begin{array}{l}\text { Croati } \\
\text { a } \\
\end{array}$ & $\begin{array}{l}74, \\
8 \\
\end{array}$ & $\begin{array}{l}69, \\
3 \\
\end{array}$ & 61 & $\begin{array}{l}60, \\
9 \\
\end{array}$ & $\begin{array}{l}63 \\
8 \\
\end{array}$ & $\begin{array}{r}70 \\
, 3 \\
\end{array}$ & $3^{7}$ & $\begin{array}{r}7 \\
6,4 \\
\end{array}$ & $\begin{array}{r}7 \\
4,5 \\
\end{array}$ & 74,6 \\
\hline $\begin{array}{l}\text { Czech } \\
\text { ia }\end{array}$ & $\begin{array}{l}467 \\
, 6 \\
\end{array}$ & $\begin{array}{l}851 \\
8 \\
\end{array}$ & $\begin{array}{c}57 \\
7,3 \\
\end{array}$ & & 355 & $\begin{array}{r}29 \\
4,7 \\
\end{array}$ & $\begin{array}{r}3 \\
06,2 \\
\end{array}$ & $\begin{array}{c}2 \\
59^{2} \\
\end{array}$ & $\begin{array}{r}2 \\
54,4 \\
\end{array}$ & $\begin{array}{l}286, \\
2\end{array}$ \\
\hline $\begin{array}{l}\text { Eston } \\
\text { ia }\end{array}$ & 32 & $\begin{array}{l}94 \\
9 \\
\end{array}$ & $\begin{array}{l}95, \\
9 \\
\end{array}$ & 53 & & $\begin{array}{l}97 \\
, 5 \\
\end{array}$ & $\begin{array}{r}1 \\
32,2 \\
\end{array}$ & $\begin{array}{r}1 \\
42,5 \\
\end{array}$ & $\begin{array}{r}1 \\
90,4 \\
\end{array}$ & $\begin{array}{l}111, \\
2\end{array}$ \\
\hline $\begin{array}{l}\text { Hung } \\
\text { ary }\end{array}$ & $\begin{array}{l}328 \\
, 7 \\
\end{array}$ & $\begin{array}{l}435 \\
, 5 \\
\end{array}$ & $\begin{array}{l}45 \\
5,2 \\
\end{array}$ & 428 & 374 & $\begin{array}{r}60 \\
6,5 \\
\end{array}$ & $\begin{array}{r}3 \\
41,5 \\
\end{array}$ & $\begin{array}{r}3 \\
19,3 \\
\end{array}$ & $\begin{array}{r}6 \\
54,3 \\
\end{array}$ & $\begin{array}{r}- \\
785,2\end{array}$ \\
\hline Latvia & 45 & 73 & $\begin{array}{l}69, \\
3\end{array}$ & $\begin{array}{l}63, \\
7\end{array}$ & 70 & $\begin{array}{l}86 \\
, 3 \\
\end{array}$ & $\begin{array}{r}9 \\
3,1 \\
\end{array}$ & $\begin{array}{r}1 \\
01,9 \\
\end{array}$ & $\begin{array}{r}1 \\
02,3 \\
\end{array}$ & $\begin{array}{l}105, \\
5\end{array}$ \\
\hline
\end{tabular}




\begin{tabular}{|c|c|c|c|c|c|c|c|c|c|c|}
\hline $\begin{array}{l}\text { Lithu } \\
\text { ania }\end{array}$ & $\begin{array}{l}37, \\
8\end{array}$ & $\begin{array}{l}19, \\
3\end{array}$ & 29 & $\begin{array}{r}- \\
12,4\end{array}$ & $\begin{array}{l}22, \\
5\end{array}$ & $\begin{array}{l}73 \\
, 6\end{array}$ & $\begin{array}{r}7^{7} \\
3,6\end{array}$ & $\begin{array}{r}8 \\
8,8\end{array}$ & $7^{6}$ & 51,5 \\
\hline $\begin{array}{l}\text { Polan } \\
\text { d }\end{array}$ & $\begin{array}{l}23, \\
6\end{array}$ & $\begin{array}{l}33, \\
9\end{array}$ & $\begin{array}{l}14, \\
8\end{array}$ & $\begin{array}{r}- \\
36,6\end{array}$ & $\begin{array}{c}- \\
1843,4\end{array}$ & $\begin{array}{r}- \\
150,2\end{array}$ & $\begin{array}{r}2 \\
64\end{array}$ & $\begin{array}{r}2 \\
89,7\end{array}$ & $\begin{array}{r}5 \\
24,2\end{array}$ & $\begin{array}{r}- \\
86,7\end{array}$ \\
\hline $\begin{array}{l}\text { Roma } \\
\text { nia }\end{array}$ & $\begin{array}{l}69, \\
7\end{array}$ & $\begin{array}{l}62, \\
1 \\
\end{array}$ & $\begin{array}{l}61, \\
4\end{array}$ & 54 & $\begin{array}{l}61, \\
7\end{array}$ & 57 & $6^{7}$ & $\begin{array}{r}6 \\
6,7 \\
\end{array}$ & $\begin{array}{r}6 \\
1,4 \\
\end{array}$ & 65,4 \\
\hline $\begin{array}{l}\text { Slove } \\
\text { nia }\end{array}$ & $\begin{array}{r}- \\
373,2\end{array}$ & $\begin{array}{r}- \\
237,1\end{array}$ & $\begin{array}{r}- \\
241,6\end{array}$ & $\begin{array}{c}- \\
3689,4\end{array}$ & $\begin{array}{l}407 \\
, 7\end{array}$ & $\begin{array}{r}28 \\
3,6\end{array}$ & $\begin{array}{r}2 \\
26\end{array}$ & $\begin{array}{l}2 \\
05\end{array}$ & $\begin{array}{c}2 \\
20\end{array}$ & 268 \\
\hline
\end{tabular}

Source: authors' own calculations based on data-series from UNCTAD database

\section{Data, Methodology and Empirical Results}

Statistics of the data series we have worked with for total FDI stock inward, total exports of GDP and total imports of GDP are presented below in Table 11. FDI as share of GDP is the most volatile series, while import as share of GDP is the least volatile among these three analysed series. Skewness alludes the tendency of a distribution that determines its symmetry about the mean. The graphs of those three analysed series show the symmetry from the mean of the dataset.

Kurtosis means the measure of the respective sharpness of the curve, in the frequency distribution. It ascertains the way observations are clustered around the centre of the distribution. Total exports and total imports data are highly dispersed against the mean of those data series, while FDI stock shows a normal distribution.

Table 11. Descriptive statistics of total FDI stock as share of GDP (FDI_GDP), total exports as share of GDP (EXP_GDP) and total imports as share of GDP (IMP_GDP)

\begin{tabular}{|l|c|c|c|}
\hline & FDI_GDP & EXP_GDP & IMP_GDP \\
\hline Mean & 54.42636 & 65.08182 & 63.38364 \\
\hline Median & 52.55000 & 66.45000 & 65.70000 \\
\hline Maximum & 94.80000 & 96.10000 & 94.00000 \\
\hline Minimum & 22.20000 & 26.00000 & 32.40000 \\
\hline Std. Dev. & 18.76473 & 17.73506 & 15.12512 \\
\hline Skewness & 0.377787 & -0.221362 & -0.148967 \\
\hline Kurtosis & 2.185970 & 1.929448 & 1.994669 \\
\hline
\end{tabular}

Source: E-views estimations

We have tested FDI stock of GDP, total exports of GDP and total imports of GDP for unit root with ADF-Fisher individual root tests and we found out that all of them are $\mathrm{I}(\mathrm{O})$. According to the Granger causality tests, the null hypothesis is rejected, thus there is a bi-directional causality between total FDI stock and total exports. There is a bidirectional causality between total FDI and total imports, and a bi-directional causality between total exports and total imports (Table 12).

Table 12. Granger causality tests between total FDI stock of GDP, total exports of GDP and total imports of GDP

\begin{tabular}{|c|c|c|}
\hline & F-Statistic & Prob. \\
\hline EXP_GDP does not Granger Cause FDI_GDP & 0.80985 & 0.4484 \\
\hline FDI_GDP does not Granger Cause EXP_GDP & 0.19704 & 0.8215 \\
\hline IMP_GDP does not Granger Cause FDI_GDP & 0.54194 & 0.5837 \\
\hline FDI_GDP does not Granger Cause IMP_GDP & 1.16019 & 0.3185 \\
\hline IMP_GDP does not Granger Cause EXP_GDP & 0.15474 & 0.8569 \\
\hline EXP_GDP does not Granger Cause IMP_GDP & 8.58431 & 0.1104 \\
\hline
\end{tabular}

Source: E-views estimations

Null Hypothesis: No causality

We have estimated a VAR for each variable: total FDI inward stock of GDP, total exports of GDP and total imports of GDP. VAR model is a multi-equation system where all the variables $\left(\mathrm{Y}_{\mathrm{t}}\right)$ are treated as endogenous (dependent) and are expressed according to their lags $\left(\mathrm{Y}_{\mathrm{t}-1}\right)$, constant term $(\mathrm{a})$ and error term $\left(\varepsilon_{\mathrm{t}}\right)$ (Equation 1).

$$
\mathrm{Y}_{\mathrm{t}}=\mathrm{a}+\mathrm{A} 1 \mathrm{Y}_{\mathrm{t}-1}+\mathrm{A} 2 \mathrm{Y}_{\mathrm{t}-2}+\ldots+\mathrm{ApY} \mathrm{t}_{\mathrm{t}-\mathrm{p}}+\varepsilon_{\mathrm{t}}
$$

We can see from the results that total FDI stock is positively correlated with total exports and total imports. The coefficients are higher for the correlation between total FDI stock and total imports. Total imports are positively 
correlated with total exports. Exports with 1 lag are positively correlated with total imports, but exports with 2 lags are negatively correlated with total imports (Table 13).

Table 13. VAR estimations for FDI stock of GDP, total exports of GDP and total imports of GDP

\begin{tabular}{|c|c|c|c|}
\hline & EXP_GDP & FDI_GDP & IMP_GDP \\
\hline EXP_GDP(-1) & 1.533018 & 0.526946 & $(0.16575)$ \\
\hline & $(0.17248)$ & $(0.42563)$ & {$[3.72346]$} \\
\hline EXP_GDP(-2) & {$[8.88789]$} & {$[1.23803]$} & -0.425087 \\
\hline & 0.556464 & 0.429819 & $(0.15942)$ \\
\hline & $(0.16590)$ & $(0.40939)$ & {$[-2.66640]$} \\
\hline FDI_GDP(-1) & {$[3.35419]$} & {$[1.04990]$} & 0.042514 \\
\hline & 0.021866 & 0.473827 & $(0.04288)$ \\
\hline FDI_GDP(-2) & $(0.04462)$ & $(0.11011)$ & {$[0.99147]$} \\
\hline & {$[0.49003]$} & {$[4.30312]$} & 0.042956 \\
\hline & 0.015501 & 0.459864 & $(0.04224)$ \\
\hline & $(0.04396)$ & $(0.10848)$ & {$[1.01686]$} \\
\hline & {$[0.35260]$} & {$[4.23912]$} & 0.862730 \\
\hline & 0.084871 & 0.394955 & $(0.15862)$ \\
\hline & $(0.16507)$ & $(0.40734)$ & {$[5.43881]$} \\
\hline & {$[0.51415]$} & {$[0.96960]$} & 0.109786 \\
\hline & 0.083194 & -0.253768 & $(0.15645)$ \\
\hline & $(0.16281)$ & $(0.40175)$ & {$[0.70173]$} \\
\hline
\end{tabular}

Statistics of the data series we have worked with in analysing the manufacturing sectors are presented below in Table 14. FDI stock in the manufacturing sectors as share of total FDI stock inward is the least volatile series, while exports of manufactured goods of total exports is the most volatile among these three analysed series. The graphs of all these three analysed series show the symmetry from the mean of the dataset. FDI in the manufactured sectors are highly dispersed against the mean of those data series, while exports and imports of the manufactured goods shows a normal distribution.

Table 14. Descriptive statistics of FDI stock in the manufacturing sectors of total FDI inward stock (FDI_MANUF), exports of the manufactured goods of total exports (EXP_MANUF) and imports of manufactured goods of total imports (IMP_MANUF)

\begin{tabular}{|l|c|c|c|}
\hline & FDI_MANUF & EXP_MANUF & IMP_MANUF \\
\hline Mean & 24.89000 & 78.94545 & 74.63182 \\
\hline Median & 27.40000 & 81.00000 & 75.85000 \\
\hline Maximum & 41.90000 & 113.9000 & 102.5000 \\
\hline Minimum & 10.80000 & 50.20000 & 50.70000 \\
\hline Std. Dev. & 8.078491 & 16.07747 & 10.89848 \\
\hline Skewness & -0.332044 & -0.053645 & -0.046003 \\
\hline Kurtosis & 1.773819 & 2.223131 & 2.777547 \\
\hline
\end{tabular}

Source: E-views estimations

We have tested FDI stock in the manufacturing sectors of total FDI stock, the exports of manufactured goods of total exports of goods and total imports of manufactured goods of total imports of goods for unit root with ADF-Fisher individual root tests and we found out that all of them are I(1). According to the Granger causality tests, there are bidirectional causality between FDI stock in the manufacturing sectors of total FDI stock, exports of the manufactured goods of total goods exports and imports of manufactured goods of total goods imports (Table 15). 
Table 15. Granger causality tests between total FDI stock in the manufacturing sectors of total FDI stock inward, the exports of manufactured goods of total exports of goods and the imports of manufactured goods of total imports of goods

\begin{tabular}{|l|c|c|}
\hline & F-Statistic & Prob. \\
\hline EXP_MANUF does not Granger Cause FDI_MANUF & 2.14493 & 0.1235 \\
\hline FDI_MANUF does not Granger Cause EXP_MANUF & 0.08483 & 0.9187 \\
\hline IMP_MANUF does not Granger Cause FDI_MANUF & 1.40286 & 0.2517 \\
\hline FDI_MANUF does not Granger Cause IMP_MANUF & 0.28328 & 0.7540 \\
\hline IMP_MANUF does not Granger Cause EXP_MANUF & 1.69263 & 0.1903 \\
\hline EXP_MANUF does not Granger Cause IMP_MANUF & 1.83488 & 0.1660 \\
\hline
\end{tabular}

Source: E-views estimations

Null Hypothesis: No causality

We have tested those three variables for co-integration, using Kao co-integration test. According to the results of the estimations, there is not a co-integration between total FDI stock in the manufacturing sectors, exports of the manufactured goods and imports of the manufactured goods (Table 16).

Table 16. Kao co-integration test for FDI stock inward in the manufacturing sectors of total FDI stock inward, exports of the manufactured goods of total exports of goods and imports of manufactured goods of total imports of goods

$\mathrm{ADF}$

\begin{tabular}{|c|c|}
\hline t-Statistic & Prob. $^{*}$ \\
-2.522005 & 0.0058 \\
\hline
\end{tabular}

Source: E-views estimations

*Null Hypothesis: No cointegration at a significance level of 0,01

As a consequence of no-cointegration for these series that are $\mathrm{I}(1)$ we have estimated a VAR using the first differences of these three series. We can see from the results that total FDI stock in the manufacturing sectors is positively correlated with exports of the manufactured goods and negatively correlated with imports of the manufactured goods. The imports of the manufactured goods are positively correlated with the exports of the manufactured goods. Exports of the manufactured goods with $1 \mathrm{lag}$ are negatively correlated with imports of the manufactured goods, but exports of the manufactured goods with 2 lags are positively correlated with imports of the manufactured goods. Previous lags of the exports of the manufactured goods are negatively correlated with the actual trend of the exports of the manufactured goods (Table 17).

Table 17. VAR estimations for FDI stock in the manufacturing sectors of total FDI stock, exports of manufactured goods of total exports of goods and imports of manufactured goods of total imports of goods

\begin{tabular}{|c|c|c|c|}
\hline & DEXP_MANUF & DFDI_MANUF DIMP_MANUF \\
\hline DEXP_MANUF(-1) & -0.471846 & 0.004125 & -0.251884 \\
\hline & $(0.19483)$ & $(0.25348)$ & $(0.20281)$ \\
\hline & {$[-2.42180]$} & {$[0.01627]$} & {$[-1.24194]$} \\
\hline DEXP_MANUF(-2) & -0.099612 & 0.074722 & 0.076996 \\
\hline & $(0.15575)$ & $(0.20263)$ & $(0.16213)$ \\
\hline DFDI_MANUF(-1) & {$[-0.63958]$} & {$[0.36876]$} & {$[0.47491]$} \\
\hline & 0.003298 & 0.194380 & -0.040382 \\
\hline & $(0.08166)$ & $(0.10624)$ & $(0.08501)$ \\
\hline DFDI_MANUF(-2) & {$[0.04039]$} & {$[1.82959]$} & {$[-0.47505]$} \\
\hline & 0.073383 & 0.243260 & -0.114721 \\
\hline & $(0.08750)$ & $(0.11384)$ & $(0.09109)$ \\
\hline & {$[0.83864]$} & {$[2.13678]$} & {$[-1.25945]$} \\
\hline & 0.337575 & 0.157429 & 0.109954 \\
\hline & $(0.17517)$ & $(0.22790)$ & $(0.18234)$ \\
\hline & {$[1.92717]$} & {$[0.69079]$} & {$[0.60301]$} \\
\hline
\end{tabular}

\footnotetext{
Source: E-views estimations
}

According to our empirical results, we can emphasize that an increase of total FDI stock supports both the increase on the total exports and total imports, but the imports increase more, so the commercial imbalance worsen on average in DOI: $10.25103 /$ ijbesar.132.01 
the CEE region. The FDI stock in the manufacturing sectors supports the increase of the exports of the manufactured goods, but an increase of the FDI stock in the manufacturing sectors doesn't support the increase of imports, so the commercial balance of the manufactured goods improves. That is why, the CEE countries displaying high shares of FDI stock in the manufacturing sectors also display high share of exports of the manufactured goods and commercial surplus of the trade balance of the total goods and of the manufactured goods.

The positive correlation between total FDI stock and total exports is weaker than the positive correlation between the FDI stock in the manufacturing sectors and exports of manufactured goods. An increase of total FDI stock increase also the total imports, but decrease the imports of the manufactured goods and supports the improvement of the trade balance of the manufactured goods. Thus, the CEE region presents an advantage in the manufacturing sectors for their external trade and should aim to attract more FDI inward in the manufacturing sectors by designing adequate economic policies. The CEE countries should aim attracting FDI especially in the manufactured sectors that produce manufactured goods incorporating high-technology and a high value-added. This could improve the entire trade balance in all these CEE countries.

These results indicating a stronger relationship between FDI and exports in the manufacturing sectors than the relation between total FDI and total exports are in line with the findings of Popovici (2018), Damijan et al. (2014), Kostoska and Mitrevski (2008) and Camarero and Tamarit (2004). Our results also point out that FDI represent a weaker factor for exports and imports comparing with the previous developments of these two variables. This relation was also found by the above-mentioned authors. Mahmoodi and Mahmoodi (2016) found for 8 European countries until 2013 a uni-directional causality running from FDI to exports, while we have found a bi-directional causality between total FDI-exports-imports or for FDI in the manufacturing sectors and exports and imports of manufactured goods. Also, they have found that these variables are co-integrated. We have applied Kao co-integration test and the null hypothesis of no-cointegration between those variables was accepted.

\section{Conclusion}

Many FDIs were attracted in the CEE region as a result of a rapid economic growth, a low tax climate and a low labour cost comparing to other European developed countries, but this situation reversed in the last years, because of a serious tax competition worldwide and low wages, especially in the Asian countries. FDI inflows can be stimulated by expanding free trade zones, decreasing foreign trade tax and increasing security in economic and political areas in the CEE countries. The developments of FDIs inflows in the CEE region during the last years show their stronger orientation toward the service sectors and that can undermine their exports competitiveness, given the fact that the domestic investments are insufficient in many CEE countries and these investments don't benefit of large fiscal facilities as the foreign companies do. The authorities should stimulate the production of goods in all of CEE countries, granting fiscal facilities for both categories of investors, domestic or foreign. Financial constraints represent important barriers for the domestic investors to develop and for large technology spillovers from FDI to the domestic investments.,

Hungary, Poland and the Czech Republic, the top leaders of the CEE region in terms of economic development represent an example of an impressive economic growth based on exports of manufactured goods. Slovakia and Romania also made important progress on this path, but in Romania, where the most important exporter is DaciaRenault, a car factory, the poor infrastructure represent a serious barrier for a future development in the car industry. The authorities of all CEE countries should allocate more for R\&D purposes in the manufactured industrial sectors to invest more in producing goods that incorporate high-technologies and for producing goods with high-added value. The investors should also be stimulated to allocate more funds for R\&D purposes for the same aim, for producing goods with high-added value.

A direction for a future research should be testing the relation between the FDIs stock inward and exports in the sectors of manufactured goods with high added-value and incorporating high-technology for the CEE region. We should also estimate a panel regression to stress the impact of FDI on imports and exports of manufactured goods, but also the impact of taxation, real GDP growth, labour productivity and labour cost on the exports and imports, because these also represent important factors in attracting FDI inflows, supporting exports growth and improving the trade balance situation.

\section{Acknowledgement}

The present study has been presented at the $12^{\text {th }}$ International Conference "Economies of the Balkan and Eastern European Countries", EBEEC 2020, that has been online held in Opatija, Croatia from May $29^{\text {th }}$ to $31^{\text {th }} 2020$ (http://ebeec.ihu.gr). 


\section{References}

Anghelache C., Jweida I.J., Popovici M., Stanciu E. (2016) Analysing the correlation between FDI and export, Revista Română de Statistică - Supliment nr. 10, pp.71-77.

Borensztein E., De Gregorio J., Lee Jong-Wha. (1998) How Does Foreign Direct Investment Affect Growth?, Journal of International Economics, vol. 45, pp. 115-135.

Bouras H. and Raggad B. (2015) Foreign Direct Investment and Exports: Complementarity or Substitutability An Empirical Investigation, International Journal of Economics and Financial Issues, 5(4), pp. 933-941.

Camarero, M. and Tamarit, C., 2004. Estimating exports and imports demand for manufactured goods: The role of FDI, Review of World Economics, 140(3), pp. 347-375.

Damijan, J.P. Rojec, M. and Ferjančič, M., 2008. Growing export performance of transition economies: EU market access versus supply capacity factors, LICOS Discussion Paper Series 202/2008.

Haruchi, Smeet, \& Chen. (2017). The Importance of Manufacturing in Economic Development: Has This Changed? World Development, 93, issue C, pp. 293-315.

Jevcak, A., Setzer, R., Suardi, M. (2010) Determinants of Capital Flows to the New EU Member States before and during the Financial Crisis, European Commission, Economic Papers.

Kaminski, B. (2000) How accession to the European Union has affected external trade and foreign direct investment in Central European economies, World Bank, Washington DC, pg. 19.

Karanja Geoffrey (2019) FDI and the Growth of Manufacturing Sector Output in China, available at https://soapboxie.com/economy/Foreign-Direct-Investment-FDI-and-the-Growth-of-Manufacturing-Sector-Output-in-China [accessed on 10th March 2020].

Kersan-Skabic, I., \& Zubin, C. (2009). The influence of foreign direct investment on the growth of GDP, on employment and on export in Croatia. Ekonomski Pregled, 60, pp. 119-151.

Kostoska O. and Mitrevski P. (2008) Estimating the FDI impact on economic growth and export performances of the European economies in transition, Revista Tinerilor Economisti, 1(11), pp.115-126.

Krstić, M. (2014). Rational choice theory and addiction behaviour. Market-Tržište, 26(2), 163-177.

Lovrinčević, Ž.; Buturac, G.; Marić Z. (2004) Priljev inozemnog kapitala - utjecaj na domaće investicije i strukturu robne razmjene, Ekonomski pregled, 55(11-12), pp. 894-934.

Mahmoodi M. and Mahmoodi E. (2016) Foreign direct investment, exports and economic growth: evidence from two panels of developing countries, Economic Research, 29(1), pp. 938-949.

Mishal Z., Abulaila Z., (2007) The Impact of Foreign Direct Investment and Imports on Economic Growth: The Case of Jordan, Journal of Economic and Administrative Sciences, 23(1), pp.1 - 31.

Mitić B. (2009) Performanse stranih direktnih investicija i uticaj na privrede Evropskih zemalja u tranziciji - pozicija Rrepublike Srbije, Ph.D. Thesis, FTB, Univerzitet "Alfa", Beograd, pp.131-132.

Mitic B. and Ivic M. (2016) The impact of foreign direct investment on export performance: case of European transition economies, International Journal of Management Production, 7(3), pp. 771-785.

Popovici O.C. (2018) The impact of FDI on EU export performance in manufacturing and services. A dynamic panel data approach, Romanian Journal of Economic Forecasting, XXI (1), pp. 108-123.

Raeskyesa D.G.S. and Suryandaru R.A. (2020) Competitiveness and FDI Inflows in ASEAN Member Countries, International Journal of Business and Economic Sciences Applied Research, 13(1), pp. 14-20.

Sekkat K. (2015) Manufactured Exports and FDI. In: Ayadi R., Dabrowski M., De Wulf L. (eds) Economic and Social Development of the Southern and Eastern Mediterranean Countries. Springer, Cham.

Selimi N., Reçi K., Sadiku L. (2016) The Impact of Foreign Direct Investment on the Export Performance: Empirical Evidence for Western Balkan Countries, International Review, 6(1), pp. 57-66.

UNCTAD (2002), World Investment Report (2002), New York: United Nations.

UNCTAD (1999) World Investment Report 1999, New York and Geneva.

Zaman Gh., Vasile V., Matei M, Croitoru C., Enescu G. (2011) Some challenging macroeconomic aspects of FDI in Romania, Romanian Journal of Economics, vol. 33, pp. 21-58.

This is an Open Access article distributed under the terms of the Creative Commons Attribution Licence

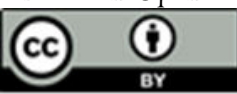

